\section{How can I manage compassion fatigue?}

\section{Most doctors will develop some form of compassion fatigue during their career. Abi Rimmer hears from experts on how to deal with it}

Abi Rimmer

\section{Set boundaries between home and work}

Shirley Remington, vice chair of the National Association of Clinical Tutors, says, "A good way to start to manage compassion fatigue is to be aware of how to recognise it and use simple routines that will lower the risk of developing it.

"Compassion fatigue, also known as secondary traumatic stress, arises from repeated exposure to the suffering of others. It often occurs suddenly which makes it different from burnout. Symptoms include a loss of empathy and feelings of guilt as a result. Increased irritability, frustration, and fatigue also feature, with a dread of work and interacting with patients. Those who are most empathetic are, ironically, most at risk.

"To lower the risk of developing compassion fatigue we can build our wellbeing through simple actions such as improving sleep patterns, taking regular exercise, exploring new things, and keeping social contact. Meditation and mindfulness are supportive, but walking, reading, or watching a box set will work just as well.

"Setting boundaries between home and work is also helpful-try to consciously park the problems from work when you leave for the day and only re-engage when you return to work. Writing down or recalling three good things that have happened at work-a gratitude diary-may help too.

"During the working day, taking breaks and eating and drinking regularly are important. Evidence shows that regular breaks enhance performance and this is particularly so later in shifts. Encouraging opportunities to talk about worries and concerns routinely can decrease the incidence of compassion fatigue.

“Taking annual leave, maintaining a life outside work, and getting into good habits within work may not be enough, so do use professional support if you need to-it's alright not to be alright."

\section{We all need to refuel ourselves}

Charlotte Squires, specialty registrar in geriatric medicine, says, "Medicine is an emotive job. Many of our encounters involve meeting people when something worrying or stressful has happened. This can be challenging, but more so when there are additional factors such as restrictions on visiting or lengthy waiting lists over which we exert little control. While the patient should always come first, we also need to safeguard our own emotional wellbeing to achieve this.
"I'm aware that when I'm tired, overworked, and perhaps have additional complexities to navigate in my personal life, I can sometimes start to feel compassion fatigue. Being aware that we only have so much fuel in our emotional tanks is a metaphor I find helpful. It helps me to consider what I need to do to recharge and refuel before the warning light comes on-this might mean organising annual or study leave, or perhaps just seeking out someone trusted to speak to. While it sounds trite, keeping a list of good things every week also helps me to maintain perspective-even in the most harried weeks, I can always find something positive to consider.

"I also find it helpful to remind myself that our job is a hard one-would a member of the public think that telling someone they have a new advanced cancer, or that someone's mother has had a severe stroke and may not survive, is easy? I don't think so. These tasks should feel difficult, because they are; when they become just another task on a list it's a sign that we need to take stock. As I become more senior, I'm aware that I have a responsibility to junior colleagues to be open about this as none of us are immune. Similarly, hearing senior colleagues describe their concerns can be affirming."

\section{Take time away from work}

Satpal Singh Shekhawat, medical director, NHS North Lincolnshire, says, "Compassion fatigue is seen among a diverse range of health professions; prevalence is variable with no consistency with demographic, personal, or professional variables. ${ }^{1}$ In the current working environment, where workload is immense and physical fatigue is setting in, emotionally tired.

"Compassion fatigue can be identified by self-reflection or through observation by colleagues. In primary care settings, for example, reception teams are well placed to spot the signs. They often will notice that a particular doctor or nurse is not their usual self and appears stressed at work.

"This soft intelligence within close knit working environments can be helpful in identifying a colleague in distress, and then measures can be put in place to help that person. There are also tools such as the professional quality of life scale that can be used to identify compassion fatigue. ${ }^{1}$

"There are many ways of tackling compassion fatigue but identifying and acknowledging it is the most important step. Personal reflective debrief after an adverse event and discussion of the event in a blame healthcare professionals are becoming increasingly 
free environment have been found to improve resilience. ${ }^{2}$

"My personal approach is to plan and take dispersed annual leave. This allows me to have timely, frequent breaks where I switch off from work and focus on family, hobbies, exercise, and reading. I've noticed that having regular holidays provides me with time away from an intense work environment-allowing for the mind, body, and soul to recover-and improves my productivity when I return to work."

\section{Put your wellbeing first}

Megan Howarth, consultant in palliative medicine at University Hospitals Dorset, says, “I probably shouldn't admit to experiencing compassion fatigue, as palliative medicine consultants are supposed to be unwaveringly compassionate at all times. I wonder, however, if the more compassionate and empathetic you are, the more likely you are to experience compassion fatigue because you put your own needs last. This can slowly wear you down.

"The better I get at tuning in to my own state of wellbeing and doing what I need to do to take care of myself, the more compassion I feel towards my patients and colleagues. Sometimes, the more you give, the more you lose yourself. I have learnt that the more effort I put into looking after myself the better I am at my job. I also enjoy it more.

"Like a lot of doctors, I've spent most of my career with some degree of burnout. Compassion fatigue is certainly a useful red flag for me that something needs to change. Wellbeing is a bit of a buzzword at the moment, associated with a lot of empty gestures and talk of the dreaded ' $r$ ' word: resilience. For me, wellbeing means being kind to myself, being realistic about what I can achieve, and making a bit of effort to do the things that I know restore me.

"Sometimes it can feel like the way to deal with compassion fatigue is to feel bad about it and so make more effort to show compassion to others; this just makes things worse. As unnatural as it feels, putting the effort into showing compassion to yourself is the best option in the long term.”

\section{Practise looking after yourself}

Jan Smith, chartered psychologist at Healthy You and clinical lead at Make Birth Better, says, "Compassion fatigue is profound mental and physical exhaustion characterised by an erosion of our empathy, compassion, and hope for others and ourselves, which accumulates over time.

"You may feel more distant or irritated with your loved ones. At work, you may feel intolerant of those you work with or become cynical. It might be more subtle, however: you might behave in the right way, but be thinking, 'I've seen much worse.'

"Every doctor who cares about their patients will develop a certain amount of compassion fatigue during their career. You wouldn't expect to walk in the rain and not get wet. Equally, if you're empathically connected with those in your care, it will take its toll. Working in a team and organisation that recognises this and actively mitigates its impact is crucial.

"It's helpful to know your signs and responses to compassion fatigue. Questions to consider might be: what are the signs that my usual levels of compassion fatigue are rising? When am I more likely to experience these? What can I put in place to support myself at these times?

"Evidence based ways to manage compassion fatigue include ensuring you take breaks at work, making time for hobbies and leisure activities away from work, and having non-work friends. It also helps to routinely check in at the end of each shift about levels of compassion fatigue and openly discuss compassion fatigue within your team to normalise it.

"Like anything, the more we practise looking after ourselves, the more we have in our reserves when needed. A career in medicine can be enriching-it doesn't have to come at the cost of your mental and physical health.”

Cavanagh N, Cockett G, Heinrich C, et al. Compassion fatigue in healthcare providers: A systematic review and meta-analysis. Nurs Ethics 2020;27:639-65. doi: 10.1177/0969733019889400 pmid: 31829113

2 Schmidt M, Haglund K. Debrief in emergency departments to improve compassion fatigue and promote resiliency. J Trauma Nurs 2017;24:317-22. doi: 10.1097/JTN.0000000000000315 pmid: 28885522 